\title{
Impact of Protein Energy Malnutrition on Outcomes of Adults With Viral Pneumonia: A Nationwide Retrospective Analysis
}

\author{
Valeria P. Trelles-Garcia ${ }^{1}$, Daniela Trelles-Garcia ${ }^{2}$, Asim Kichloo ${ }^{3}$, Sairam Raghavan ${ }^{4}$, Pius E. Ojemolon ${ }^{5}$ \\ , Precious Eseaton ${ }^{6}$, Osahon N. Idolor ${ }^{6}$ \\ 1. Internal Medicine, John H. Stroger, Jr. Hospital of Cook County, Chicago, USA 2. Internal Medicine, Saint Francis \\ Hospital, Evanston, USA 3. Internal Medicine, Central Michigan University, Saginaw, USA 4. Internal Medicine, John H \\ Stroger, Jr. Hospital of Cook County, Chicago, USA 5. Anatomical Sciences, St. George's University, St. George's, GRD 6. \\ Internal Medicine, College of Medicine, University of Benin, Benin City, NGA
}

Corresponding author: Osahon N. Idolor, osahon.idolor@yahoo.com

\section{Abstract}

\section{Background}

Community-acquired pneumonia (CAP) is associated with significant morbidity and mortality. Viral organisms have been identified as the causal pathogen in approximately $20 \%$ of CAP. Nutritional status plays an important role in the response to pneumonia. This study aims to identify whether protein energy malnutrition (PEM) is an independent risk factor for mortality and morbidity in viral CAP.

\section{Materials and methods}

This was a retrospective cohort study involving adult hospitalizations for viral CAP in the United States using the Nationwide Inpatient Sample (NIS) database. This cohort was further divided based on the presence or absence of a secondary discharge diagnosis of PEM. The primary outcome was inpatient mortality. Secondary outcomes included the rate of mechanical ventilation among other complications.

\section{Results}

The in-hospital mortality for viral CAP was $2.22 \%$. Patients with PEM had over two-fold high adjusted odds of inpatient mortality (aOR: 2.42 , 95\% CI: 1.746-3.351, p < 0.001) compared with patients without PEM. Patients with PEM had higher adjusted odds of having septic shock (aOR: 3.34, 95\% CI: 2.158-5.160, p < 0.001). NSTEMI (aOR: 1.75, 95\% CI: 1.163-2.621, $\mathrm{p}=0.007$ ), need for mechanical ventilation (aOR: $3.13,95 \%$ CI: $2.448-4.006, p<0.001$ ), CVA (aOR: 3.49, 95\% CI: 1.687-7.220, $\mathrm{p}=0.001$ ), DVT (aOR: 2.19 , 95\% CI: 1.453 $3.295, \mathrm{p}<0.001$ ), and PE (aOR: $2.24,95 \% \mathrm{CI}: 1.152-4.357, \mathrm{p}=0.017$ ) relative to patients without PEM.

\section{Conclusion}

Review began $11 / 26 / 2020$ Review ended 12/15/2020 Published 12/25/2020

() Copyright 2020 Trelles-Garcia et al. This is an open access article distributed under the terms of the Creative Commons Attribution License CC-BY 4.0., which permits unrestricted use, distribution, and reproduction in any medium, provided the original author and source are credited.
In conclusion, coexisting PEM is associated with a higher rate of in-hospital morbidity and mortality in patients with viral CAP. Early identification and treatment of nutritional deficiencies can lead to improved outcomes and reduced costs.

Categories: Internal Medicine, Infectious Disease, Pulmonology

Keywords: viral pneumonia, protein energy malnutrition, nis, mortality, morbidity

\section{Introduction}

Community-acquired pneumonia (CAP) ranks first in hospitalizations and seventh in all-cause death in industrialized countries [1]. Viral organisms have been identified as the causal pathogen in approximately 1/5th of CAP. About 100 million cases of viral CAP occur every year among adults, with a mortality rate estimated to be between $3 \%$ and $24 \%[2,3]$. Viral pathogens that cause CAP include influenza, adenovirus, parainfluenza, respiratory syncytial virus, and human metapneumovirus. Advanced age, smoking, obesity and environmental exposures to different substances including metals, dust, and fumes have been linked to worse outcomes in patients with viral CAP $[3,4]$.

Nutritional status plays an important role in the outcome of many disease conditions [5-8]. Nutrition is postulated to be involved in the immune response to pneumonia. Protein energy malnutrition (PEM) is reportedly linked to lymphoid tissue atrophy, decreased delayed cutaneous hypersensitivity, fewer T cells, impaired secretory immunoglobulin A antibody response, decreased antibody affinity, reduced concentration and activity of complement components and phagocyte dysfunction [9]. Some studies found that PEM is associated with increased morbidity and mortality, longer length of hospital stay, and increased hospital costs $[10,11]$ in patients with viral CAP. Moreover, it was found to be an independent predictor of 2year mortality after discharge in the elderly patients [12]. However, the relatively small sample sizes of these 
studies raise concern for sample bias. The purpose of this study is to identify whether PEM is an independent risk factor for mortality and morbidity in viral CAP using population-level data.

\section{Materials And Methods \\ Design and data source}

This was a retrospective cohort study for viral CAP in the United States between January 1, 2016 and December 31, 2017. The Nationwide Inpatient Sample (NIS) databases for 2016 and 2017 were used. The NIS is a database of hospital inpatient stays derived from billing data submitted by hospitals to statewide data organizations across the United States, covering more than $97 \%$ of the U.S. population [13]. It approximates a $20 \%$ stratified sample of discharges from U.S. community hospitals, excluding rehabilitation and longterm acute care hospitals. This dataset is weighted to obtain national estimates [14]. Both the 2016 and 2017 databases were coded using the International Classification of Diseases, Tenth Revision, Clinical Modification/Procedure Coding System (ICD-10-CM/PCS).

\section{Study population}

Adult patients 18 years and above who had a principal discharge diagnosis of viral CAP including influenza due to identified novel influenza A virus with pneumonia (J09.X1), influenza due to other identified influenza virus with pneumonia (J10.0), influenza due to unidentified influenza virus with pneumonia (J11.0), and viral pneumonia, not elsewhere classified (J12) were included. Patients were excluded if they had super-imposed bacterial pneumonia or if viral pneumonia was only a secondary diagnosis. This cohort was further divided based on the presence or absence of a secondary discharge diagnosis of PEM (E43, E44.0, E44.1, E46).

\section{Outcome measures}

The primary outcome was comparing inpatient mortality among hospitalizations principally for viral CAP between PEM and non-PEM patients. Secondary outcomes in this population included odds of having a secondary discharge diagnosis of sepsis, septic shock, acute respiratory failure, acute respiratory distress syndrome, Non-ST segment elevation myocardial infarction (NSTEMI), acute kidney failure (AKI), deep vein thrombosis (DVT), pulmonary embolism (PE), cerebrovascular accident, need for mechanical ventilation, vasopressors as well as mean length of hospitalization (LOS) and mean total hospital charges (THC).

\section{Statistical analysis}

We analyzed the data using Stata ${ }^{\circledR}$ Version 16 software (StataCorp, College Station, TX). All analyses were conducted using the weighted samples for national estimates in adjunct with Healthcare Cost and Utilization Project regulations for using the NIS database. Co-morbidities were calculated as proportions of the cohort and Chi-squared test was used to compare these characteristics between subgroups. Multivariate regression analysis was done to adjust for possible confounders while calculating the primary and secondary outcomes. This method was employed in prior peer-reviewed publications [15-18]. Possible confounders were obtained from the literature review, and included variables from the validated MuLBSTA Score for viral pneumonia as well as the Pneumonia Severity Index [19,20]. A univariate screen was done to further confirm possible confounders with variables having a $p<0.2$ included in the multivariate regression analysis. A p of 0.05 was set as the threshold for statistical significance.

\section{Ethical considerations}

The NIS database lacks patient-level identifiers. Since 2012, the NIS has also removed most state level and hospital identifiers. This study was exempt from Institutional Review Board approval.

\section{Results}

\section{Patient characteristics}

The combined NIS database for 2016 and 2017 contained over 71 million weighted hospital discharges of which 89,650 satisfied the inclusion criteria for the study. These hospitalizations were for adult patients with a principal discharge diagnosis of viral CAP. Of these patients, $5.0 \%$ had a secondary diagnosis of PEM.

Table 1 details the patient and hospital characteristics of the studied cohort. Patients with PEM were significantly older ( 71.1 vs 67.8 years, $\mathrm{p}<0.001$ ). and were more likely to have comorbid history of malignancy ( 58.4 vs $37.4 \%, \mathrm{p}<0.001$ ), anemia $(43.2$ vs $23.9 \%$, p < 0.001 ), chronic obstructive pulmonary disease ( 32.1 vs $25.4 \%, \mathrm{p}<0.001$ ) and chronic kidney disease ( 20.2 vs $16.6 \%, \mathrm{p}=0.006$ ) compared to patients without PEM. However, patients with PEM had significantly less proportion of co-morbid hypertension (34.7 vs $41.7 \%, \mathrm{p}<0.001)$ and diabetes $(24.5$ vs $31.9 \%, \mathrm{p}<0.001)$. There was no significant difference in past or current use of tobacco between the subgroups. 


\section{Cureus}

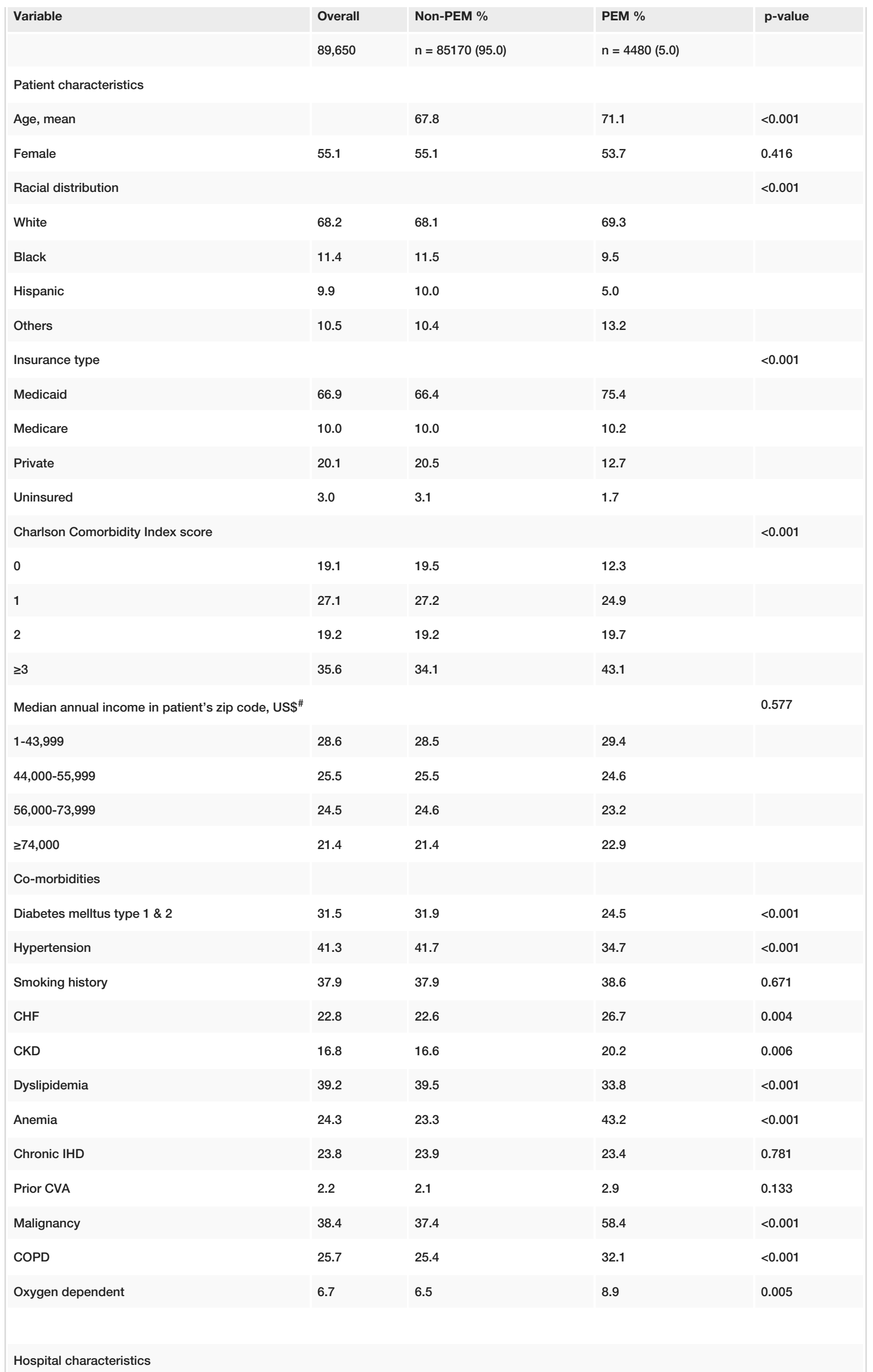




\section{Cureus}

Hospital region

Northeast

Midwest

South

West

Hospital bed size

Small

Medium

Large

Urban location

Teaching hospital

\section{7}

25.0

35.3

21.0

22.8

27.8

49.4

87.7

62.3
0.002

17.2

30.1

30.1

22.6

0.002

20.0

24.4

55.6

93.0

$<0.001$

69.2

$<0.001$

\section{TABLE 1: Patient and hospital characteristics of hospitalizations for viral pneumonia by PEM.}

\#: for 2017. CHF: congestive heart failure, CKD: chronic kidney disease, COPD: chronic obstructive pulmonary disease, CVA: cerebrovascular accident, IHD: ischemic heart disease, PEM: protein energy malnutrition, US\$: United States dollars.

\section{Primary outcome: in-hospital mortality}

The in-hospital mortality for patients principally hospitalized for viral pneumonia was $2.22 \%$. Patients with PEM had over two-fold high adjusted odds of inpatient mortality (aOR: 2.42, 95\% CI: 1.746-3.351, p < 0.001) compared with patients without PEM.

\section{Secondary outcomes}

Patients with PEM had higher adjusted odds of having septic shock (aOR: 3.34, 95\% CI: 2.158-5.160, p < 0.001), NSTEMI (aOR: 1.75, 95\% CI: 1.163-2.621, $\mathrm{p}=0.007$ ), need for mechanical ventilation (aOR: $3.13,95 \%$ CI: 2.448-4.006, p < 0.001), CVA (aOR: 3.49, 95\% CI: 1.687-7.220, p=0.001), DVT (aOR: 2.19, 95\% CI: $1.453-$ $3.295, \mathrm{p}<0.001$ ), and PE (aOR: $2.24,95 \% \mathrm{CI}: 1.152-4.357, \mathrm{p}=0.017$ ) relative to patients without PEM. Patients with PEM also had significantly longer LOS as well as higher THC. Table 2 provides a summary of the clinical outcomes. 


\section{Cureus}

\begin{tabular}{|c|c|c|c|c|}
\hline Outcome & Non-PEM, \% & PEM, \% & aOR $(95 \% \mathrm{Cl})$ & p-value ${ }^{*}$ \\
\hline \multicolumn{5}{|l|}{ Primary outcome } \\
\hline In hospital mortality & 2.0 & 6.3 & $2.42(1.746-3.351)$ & $<0.001^{\star}$ \\
\hline \multicolumn{5}{|l|}{ Secondary outcomes } \\
\hline Length of stay, mean & 4.9 & 9.1 & $3.63^{\#}(2.987-4.277)$ & $<0.001^{\star}$ \\
\hline Total hospital charges, mean US\$ & 43995 & 93841 & $42591^{\#}(30879-54304)$ & $<0.001^{\star}$ \\
\hline Sepsis & 2.9 & 8.3 & $2.58(1.962-3.387)$ & $<0.001^{\star}$ \\
\hline Septic shock & 0.8 & 3.6 & $3.34(2.158-5.160)$ & $<0.001^{\star}$ \\
\hline NSTEMI & 1.7 & 4.0 & $1.75(1.163-2.621)$ & $0.007^{\star}$ \\
\hline Mechanically ventilated & 3.5 & 11.2 & $3.13(2.448-4.006)$ & $<0.001^{*}$ \\
\hline Required pressors & 2.2 & 2.8 & $1.14(0.746-1.734)$ & 0.542 \\
\hline Acute kidney failure & 17.2 & 25.9 & $1.45(1.220-1.723)$ & $<0.001^{*}$ \\
\hline Acute respiratory failure & 25.1 & 32.6 & $1.33(1.139-1.544)$ & $<0.001^{\star}$ \\
\hline ARDS & 0.8 & 1.6 & $1.78(0.990-3.188)$ & 0.054 \\
\hline Deep vein thrombosis & 1.1 & 3.4 & $2.19(1.453-3.295)$ & $<0.001^{*}$ \\
\hline Pulmonary embolism & 0.5 & 1.2 & $2.24(1.152-4.357)$ & $0.017^{\star}$ \\
\hline Cerebrovascular accident & 0.3 & 1.2 & $3.49(1.687-7.220)$ & $0.001^{\star}$ \\
\hline
\end{tabular}

TABLE 2: Clinical outcomes of hospitalizations for viral pneumonia by PEM.

*Statistically significant, \#adjusted mean difference. aOR: adjusted odds ratio, Cl: confidence interval, ARDS: acute respiratory distress syndrome, NSTEMI; non-ST segment elevation myocardial infarction, PEM: protein energy malnutrition, US\$: United States dollars.

\section{Discussion}

About $5 \%$ of adults with viral pneumonia had a secondary diagnosis of PEM. In-patient mortality was two times more likely to occur if viral pneumonia coexisted with PEM. These patients also had high odds of requiring mechanical ventilation, having septic shock, DVT, PE, had longer hospital stay, and incur higher hospital costs compared to patients without PEM.

Only 5\% of patients with viral CAP had PEM, which was significantly lower than previously reported. One study showed that the prevalence of malnutrition is as high as $40 \%$ among hospitalized patients [21], rising to $53.4 \%$ of elderly patients [12]. The low prevalence of PEM in the study population may be explained by under-diagnosis; the lack of an initial nutritional assessment in many hospitalized patients may be the driving factor. The absence of a standardized definition of PEM is another contributor. Hypoalbuminemia, hypoproteinemia, malnourishment, among others could have been used as surrogate diagnosis for PEM. The increased risk of septic shock is probably related to the detrimental effect that malnutrition has in the immune response. however, the relation between nutritional status and deep venous thrombosis or pulmonary emboli is unclear. Decreased ambulatory capacity in the setting of poor nutrition and critical illness remains a hypothesis. The association between longer length of hospitalization with higher hospital charges and PEM is well documented. This has a remarkable implication for healthcare-related costs. If malnutrition was optimally assessed, and treated, CAP economic burden could decrease significantly. Nutritional status is a proven determinant of the health trajectory and outcome following hospital discharge [12]. Yeo et al showed that despite the resolution of pneumonia, malnourished elderly people have an increased risk of future impaired muscle and respiratory function, leading to increased long-term mortality [12]. Mortality increased in proportion to the degree of malnutrition [22]. We agree that targeted risk reduction interventions based on recognizing risk factors for CAP are of primary importance in reducing CAP related death rates [23]. This study emphasizes the need to assess every patient's nutritional status on admission, particularly in the elderly. It is worth mentioning that to date, no single parameter is definitive for determining malnutrition in the elderly [24].

This study has some important limitations. The NIS uses "claims data", and ICD-10 codes were used to confirm diagnoses rather than clinical parameters $[25,26]$. NIS does not appropriately grade disease severity 
[26]. It involved a retrospective database subject to nonrandomization. Microbiologic confirmation of specific viral causes of CAP is cost-intensive with little clinical relevance, hence often not performed routinely. Sputum and blood culture-negative bacterial CAP, especially atypical CAP may be misclassified as viral CAP. NIS report data hospitalization data, rather than individual patients, hence patients with multiple hospital encounters will be captured multiple times [27,28]. NIS does not contain information on disease duration [29], hence we cannot determine if this PEM disease duration may have played a role in the outcomes of viral pneumonia hospitalizations. NIS studies do not prove causation, only association can be obtained. Nonetheless, this database provided the unique opportunity to study this cohort at a population level. Multiple centers are accounted for ensuring representation of an ample geographic area. Comprehensive literature search, use of sound methodological review techniques and an exhaustive review process have developed this piece of evidence.

\section{Conclusions}

In conclusion, coexisting PEM is associated with higher rate of in hospital morbidity and mortality in patients with viral CAP. Early identification and treatment of nutritional deficiencies can lead to improved outcomes and reduced costs.

\section{Additional Information \\ Disclosures}

Human subjects: Consent was obtained by all participants in this study. N/A issued approval N/A. Since the National Inpatient Sample contains de-identified publicly available data, IRB board approval was waived. Animal subjects: All authors have confirmed that this study did not involve animal subjects or tissue. Conflicts of interest: In compliance with the ICMJE uniform disclosure form, all authors declare the following: Payment/services info: All authors have declared that no financial support was received from any organization for the submitted work. Financial relationships: All authors have declared that they have no financial relationships at present or within the previous three years with any organizations that might have an interest in the submitted work. Other relationships: All authors have declared that there are no other relationships or activities that could appear to have influenced the submitted work.

\section{References}

1. Tokgoz Akyil F, Yalcinsoy M, Hazar A, et al.: Prognosis of hospitalized patients with community-acquired pneumonia. Pulmonology. 2018, 24:164-169. 10.1016/j.rppnen.2017.07.010

2. Ruuskanen O, Lahti E, Jennings LC, Murdoch DR: Viral pneumonia. Lancet. 2011, 377:1264-1275. 10.1016/S0140-6736(10)61459-6

3. Almirall J, Serra-Prat M, Bolíbar I, Balasso V: Risk factors for community-acquired pneumonia in adults: a systematic review of observational studies. Respiration. 2017, 94:299-311. 10.1159/000479089

4. Shaka H, Raghavan S, Trelles-Garcia VP, et al.: Predicting COVID-19 using retrospective data: impact of obesity on outcomes of adult patients with viral pneumonia. Cureus. 2020, 12:e10291. 10.7759/cureus.10291

5. Shaka H, Edigin E, Raghavan S, et al.: The obesity paradox among patients hospitalized for bacterial pneumonia: outcomes of the nationwide inpatient sample. Chest. 2020, 158:A335. 10.1016/j.chest.2020.08.332

6. Shaka H, Ojemolon PE: Impact of obesity on outcomes of patients with hip osteoarthritis who underwent hip arthroplasty. Cureus. 2020;12(10, 12:e10876. 10.7759/cureus.10876

7. Shaka H, Gomez TM: Bronchogenic malignancies and the obesity paradox: adding weight to a growing argument using the nationwide inpatient sample. Chest. 2020, 158:A1470. 10.1016/j.chest.2020.08.1326

8. Shaka H, Padilla Sorto ME, Gomez TMA, Edigin E, Xu J, Yap SET: 242-LB: the obesity paradox among patients hospitalized for diabetes and its complications: outcomes of the nationwide inpatient sample. Diabetes. 2020, 69:242. 10.2337/db20-242-LB

9. Chandra RK: Protein-energy malnutrition and immunological responses. J Nutr. 1991, 122:597-600. 10.1093/jn/122.suppl_3.597

10. Barker LA, Gout BS, Crowe TC: Hospital malnutrition: prevalence, identification and impact on patients and the healthcare system. Int J Environ Res Public Health. 2011, 8:514-527. 10.3390/ijerph8020514

11. Jensen GL: Inflammation as the key interface of the medical and nutrition universes: a provocative examination of the future of clinical nutrition and medicine. JPEN J Parenter Enteral Nutr. 2006, 30:453463. 10.1177/0148607106030005453

12. Yeo HJ, Byun KS, Han J, et al.: Prognostic significance of malnutrition for long-term mortality in community-acquired pneumonia: a propensity score matched analysis. Korean J Intern Med. 2019, 34:841849. 10.3904/kjim.2018.037

13. Edigin E, Ojemolon PE, Eseaton PO, et al.: Systemic sclerosis is associated with increased inpatient mortality in patients admitted for atrial fibrillation: analysis of the national inpatient sample [ahead of print]. J Clin Rheumatol. 2020, 10.1097/RHU.0000000000001543

14. Edigin E, Ojemolon PE, Eseaton PO, et al.: Rheumatoid arthritis patients have better outcomes when hospitalized for ischemic stroke: analysis of the national inpatient sample [ahead of print]. J Clin Rheumatol. 2020, 10.1097/RHU.0000000000001563

15. Ojemolon PE, Shaka H, Edigin E, et al.: Impact of diabetes mellitus on outcomes of patients with knee osteoarthritis who underwent knee arthroplasty: an analysis of the nationwide inpatient sample. Cureus. 2020, 12 :e8902. 10.7759/cureus.8902

16. Edigin E, Shaka H, Eseaton P, et al.: Rheumatoid arthritis is not associated with increased inpatient mortality in patients admitted for acute coronary syndrome. Cureus. 2020, 12:e9799. 10.7759/cureus.9799 
17. Asemota I, Shaka H, Ehizogie E, et al.: TCT CONNECT-78 coexisting protein energy malnutrition is associated with increased mortality in patients admitted for transcatheter aortic valve replacement: analysis of the national inpatient sample. J Am Coll Cardiol. 2020, 76:B34-B35. 10.1016/j.jacc.2020.09.092

18. Shaka H, Raghavan S, Edigin E: Is the presence of diabetes mellitus a poor prognostic factor during hospitalizations for bacterial pneumonia? An analysis of the nationwide inpatient sample. Chest. 2020, 158:A334. 10.1016/j.chest.2020.08.331

19. Guo L, Wei D, Zhang X, et al.: Clinical features predicting mortality risk in patients with viral pneumonia: the MuLBSTA score. Front Microbiol. 2019, 10:2752. 10.3389/fmicb.2019.02752

20. Fine MJ, Auble TE, Yealy DM, et al.: A prediction rule to identify low-risk patients with community-acquired pneumonia. N Engl J Med. 1997, 336:243-250. 10.1056/NEJM199701233360402

21. Kopelman P, Lennard-Jones J: Nutrition and patients: a doctor's responsibility . Clin Med. 2002, 2:391-394. 10.7861/clinmedicine.2-5-391

22. Rodríguez-Pecci MS, Carlson D, Montero-Tinnirello J, Parodi RL, Montero A, Greca AA: Nutritional status and mortality in community acquired pneumonia [Article in Spanish]. Medicina. 2010, 70:120-126.

23. Almirall J, Bolíbar I, Serra-Prat M, et al.: New evidence of risk factors for community-acquired pneumonia: a population-based study. Eur Respir J. 2008, 31:1274-1284. 10.1183/09031936.00095807

24. Harris D, Haboubi N: Malnutrition screening in the elderly population . J R Soc Med. 2005, 98:411-414. 10.1258/jrsm.98.9.411

25. Jamal S., Khan M.Z., Kichloo A., et al.: The effect of atrial fibrillation on inpatient outcomes of patients with acute pancreatitis: a two-year national inpatient sample database study. J Innov Cardiac Rhythm Manage. 2021, 1-6. 10.19102/icrm.2021.120102

26. Edigin E, Akuna E, Asemota I, et al.: Rheumatoid arthritis does not negatively impact outcomes of patients admitted for atrial fibrillation. Cureus. 2020, 12 :e10241. 10.7759/cureus.10241

27. Edigin E, Eseaton P, Kaul S, et al.: Systemic sclerosis is not associated with worse outcomes of patients admitted for ischemic stroke: analysis of the national inpatient sample. Cureus. 2020, 12:e9155. 10.7759/cureus.9155

28. Edigin E, Kaul S, Eseaton PO: Analysis of hidradenitis suppurativa hospitalizations: a report from the National Inpatient Sample database (in press). J Am Acad Dermatol. 2020, 10.1016/j.jaad.2020.10.083

29. Edigin E, Ojemolon PE, Eseaton PO, et al.: Systemic sclerosis is associated with increased inpatient mortality in patients admitted for acute coronary syndrome: analysis of the national inpatient sample (ahead of print). J Clin Rheumatol. 2020, 10.1097/RHU.0000000000001634 